\title{
RAPID KECK SPECTROSCOPY OF CATACLYSMIC VARIABLES
}

\author{
Warren Skidmore, ${ }^{1}$ Keith Horne, ${ }^{2}$ Kovin Pearson ${ }^{3}$ Richard Gomer. ${ }^{4}$ Kieran O'Brien, ${ }^{5}$ and Ber ()ke ${ }^{6 j}$
}

RESUMEN

Presentamos un análisis de Espectroscopía Rápida de Kerk do las CVs AM Her (polar) y SS-Cyg (nova enana). Descomponemos los espectros en componentes constantes y variables c irlentificamos diferentes tipos de variabilidad en AM Her con diferentes escalas características de timpo. Se aislaron la componente variable del flujo del disco de acreción y las características observacionales de una pequeña fulguración en SS ('vg.

\section{ABSTRAC'T}

Wr present an analysis of Rapid Keck Spectroscopy of the ("Vs A.Y Her (polar) and SS ('rge (rwarf nowa). We decompose the spectra into constant and variable components and identify different $1, y$ pes of variability in AM Her with different characteristic timescales. The variable flickering connponent of the arcretion disc flux and the observational characteristics of a small flare in s's Cyg are isolated.

Key Words: NOVAE, CATACLYSMIC VARIABLES - STARS: INDIVIDUAL (AM HER, SS CYG)

\section{INTRODUCTION}

AM Her is the prototypical Polar system - the accretion stream threads onto the white dwarf magnetosphere, accrcting down onto one of the magnetic poles. The white dwarf is locked into corotation with the binary. Variability occurs on a variety of timescales, ellipsoidal modulation on hour timescales, flickering from the threading and impact regions on second timescales.

The dwarf nova SS Cyg has a record of visual magnitude observations stretching back over 100 years. Variability in SS Cyg arises due to orbital modulations on hour timescales and accretion disc flickering on second timescales.

A.I Her \& SS Cyg were observed during a campaign to observe LMXBs \& CVs at high time resolution with the Keck LRIS. Reports of analysis of observations from this campaign are O'Brien et al. (2001), Steeghs et al. (2001) and Skidmore et al. (2003a). These references and Skidmore et al. (2003b) describe the data reduction process and peculiar problems with this data set that were overcome during analysis. Early analysis of the AM Her and SS Cyg observations has been reported in Skidmore et al. (2003b).

The data cover $3017 \AA$ to $7989 \AA$ with $2.4 \AA$ pix dispersion and $72 \mathrm{~ms}$ integration time per spectrum

\footnotetext{
${ }^{1}$ California Institute of Technology, Pasadena.

${ }^{2}$ Physics \& Astronomy, Lniversity of St. Andrews.

${ }^{3}$ Physics \& Astronomy, Louisiana State University.

${ }^{4}$ Howard Hughes Medical Institute, Department of Biochemistry \& Cell Biology, Rice University.

${ }^{5} \mathrm{ESO} / \mathrm{Chile}$.

${ }^{6}$ Dominion Astrophysical Observatory, Victoria.
}

with no dead time. Wo caul decompose such datid into constant and variable components (Skidmore of al. 2003b). This simple decomposition is improved by filtering to suppress rapid variability. subtracting the smoothed data from the raw data and fit ting to the fast and slow components simultanounsly.

\section{AN HER}

AM Her was observed on 1998 July 2 for 16894 (orbital phases 0.388 to 0.539-Schwar\% et al. 20(5)2). All light curves have the same basic foratures (Skidnore et al. 2003b). The blue continumu and $\mathrm{H} \beta-\delta$ line light curves have slight differenes in the relative sizes of rapid variations in each light 'strve. The red continuum light curve has small short timescale differences compared to the other light curves. Power spectra of the light curves have similar slopes. The rapid uarialility is probably due to the discrete but random flow of blobs onto the pole.

The mean spectrum has strong Balnee of Hell lines. weak HeI and Balneer jump in ('nnission, typical of polars (Skidmore ot al. 2003h). The simple decomposition residuals rise around the emission lines and are broader than the R.MS spectrum e'mission lines, indicating that the decomposition fails to describe the line wing variability. Thus the line wings vary on a significantly different timescale and manner to the continumu, this difference becomes larger toward the blue (Skidmore et al. 2003b).

\subsection{Tue component erarability}

Fig. 1, the fast rariability spectrum emission lines are ied shifted and optically thin. The strength of the red-shifted fast sariable component and the 


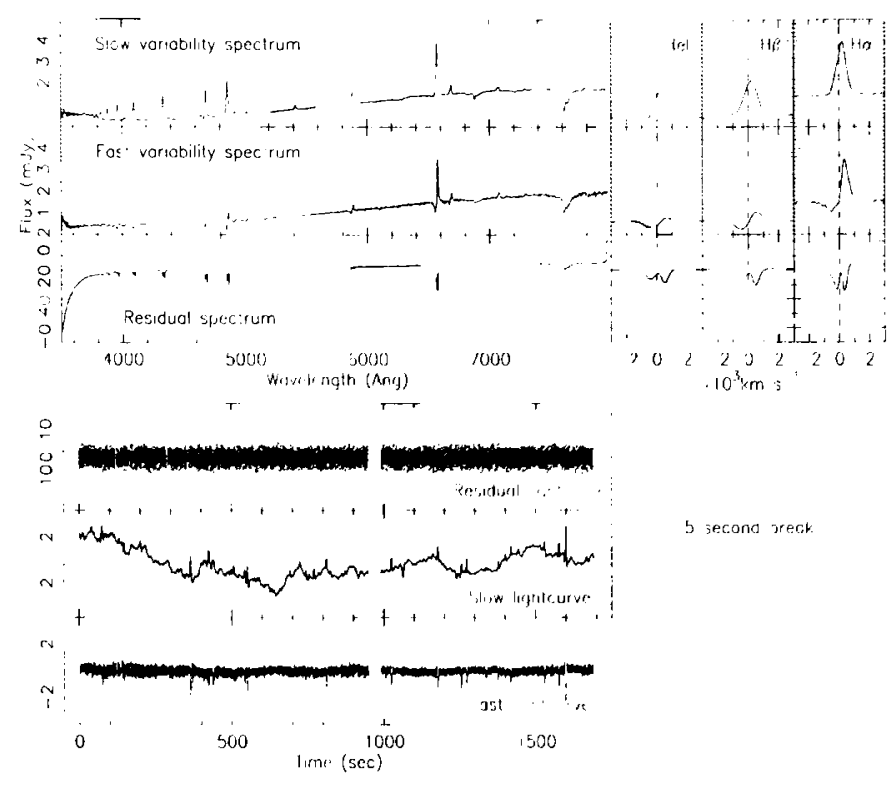

Fig. 1. Fast \& slow variability spectra \& variability light curves of AM Her, break period is 5 seconds.

amount of red shift increases as the break period decreases. The red rise in the variability spectra is most probably due to rapidly varying cyclotron flux. Emission line equivalent width in the slow variability spectrum is lower than in the mean spectrum, indicating an optically thick variability source compared to main line emitting regions.

\section{SS CYG}

SS Cyg was observed on 6 July 1998, 1044s covered orbital phases $0.828-0.872$. Light-curves of SS Cyg (Skidmore ot al. 2003b) show a general decline in flux with small amplitude variability of $\sim 0.045$ mags and a flare lasting 2.5 minutes.

\subsection{Flare light-curves}

Fig. 2 shows light-curves of the small flare in four continuum regions. The bluest Paschen continuum light-curve rises, peaks \& drops before the redder light-curves. The Balmer continuum light curve rises after but falls before the blue Paschen continuum.

\subsection{Spectra throughout flare}

Fig. 3 shows spectra obtained at points throughout the small flare (see dots in figure 2) after removing the underlying spectrum. The red continuum, Balmer continuum and high order lines rise rapidly indicating an increase in emitting region temperature, the continuum flux rises more strongly toward the blue. At mid \& late rise the emission line flux $\&$ width increases. At the peak the emission lines are narrow, the emitting material has a high optical depth, we see lots of HeII $(4686 \AA) \& \mathrm{HeI}(5876 \AA \&$

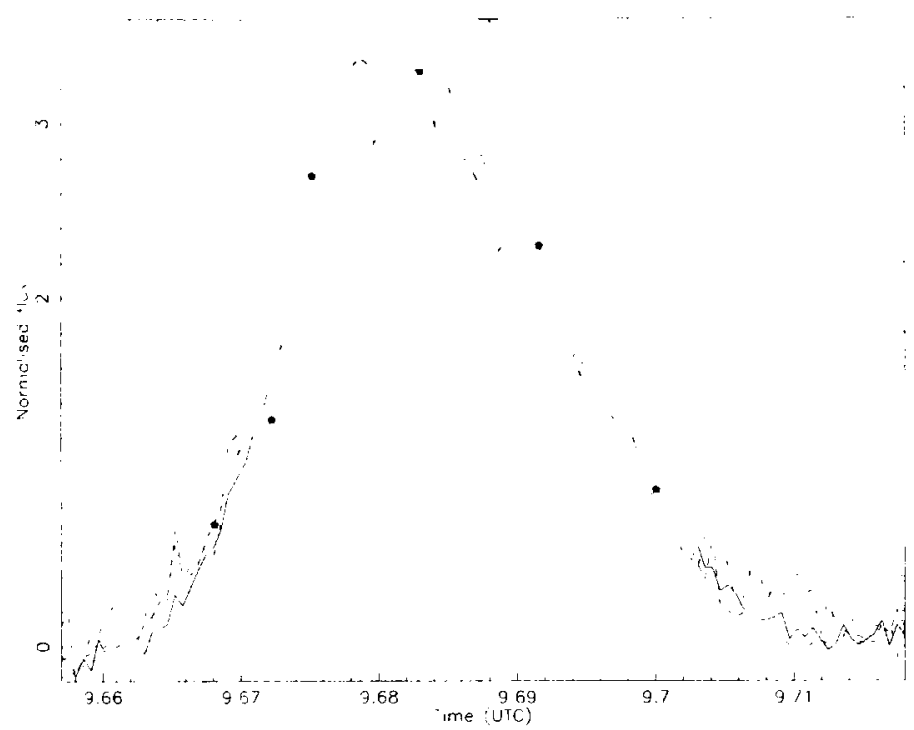

Fig. 2. Continuum light-curves of the flare, $3590 \AA$ to $3640 \AA$ (dot-dashed), $4165 \AA$ to $4285 \AA$ (dashed), $5400 \AA$ to $5600 \AA$ (solid) and $7080 \AA$ to $7560 \AA$ (dotted). Dots show the times at which spectra shown in figure 3 were measured. $\mathrm{Y}$ axis scaling is normalised.

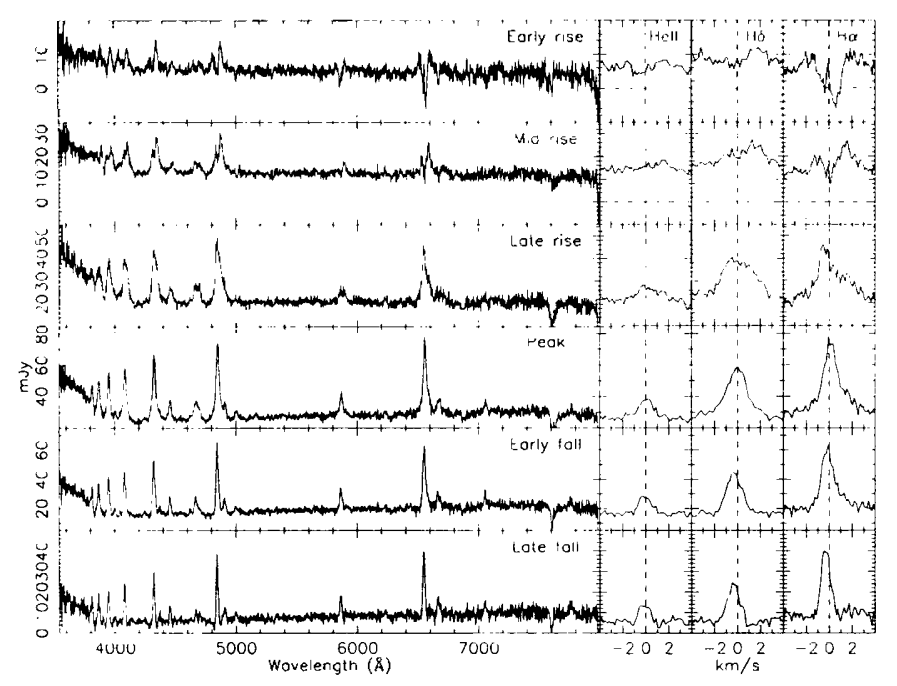

Fig. 3. Spectra at times throughout the flare.

$4472 \AA$ ) emission. Early decline shows a large decrease in emission line width. In the latter decline the Balmer jump and emission line flux \& widths fall significantly, the continuum flux becomes very red.

\subsection{Variability spectra}

Fig. 4 shows the variability spectra for various parts of the data set \& the time averaged spectrum. These show that the variability spectrum of the flare is the same as the variability spectrum of the small scale flickering. This means that the flickering probably arises due to a similar process as the flare.

\section{CONCLUSIONS}

In AM Her we have shown that there appears to be at least two distinct types of variability with 


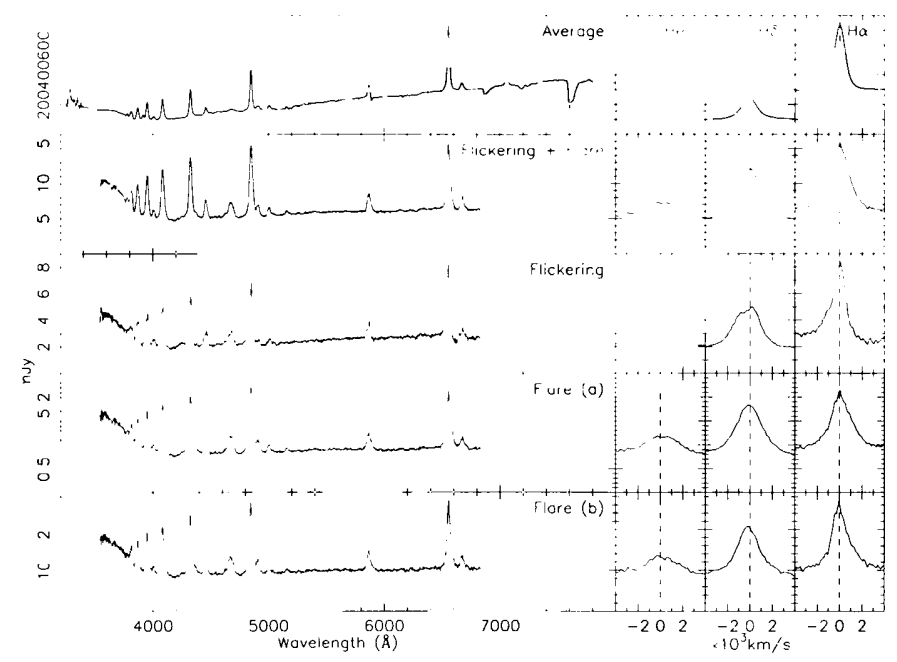

Fig. 4. Top panel shows the mean spectrum of SS Cyg. Next panel shows the variability spectrum of the complete data set after removal of a low order polynomial (to remove long period variations orbital, non-flickering). Lower panels show the variability spectra of the flickering only, the flare only and the flare after the removal of the underlying spectrum as described in section 3.2.

different spectral and temporal characteristics. Further analysis and modeling of these fast and slow variability spectra is planned.

We have extracted the variable component of the accretion flux in SS Cyg and shown that small scale flickering and larger flares appear to have a common origin. We are beginning to model the accretion disc variability spectra using the fireball model (Pearson et al. 2003, 2004).
The work presented here is incorporated in two papers to be submitted to MNRAS

Data presented herein were obtained at the W.M. Keck Observatory, which is operated as a scientific partnership among the California Institute of Technology, the University of California and the Nittional Aeronautics and Space Administration. The Observatory was made possible by the generous financial support of the W.M. Keck Foundation. Wr thank Tom Marsh for the use of his MOLLY analysis software.

\section{REFERENCES}

O'Brien K., Horne K., Boroson B., Still M., Gomer R.. Oke B., Boyd P. \& Vrtilek S., 2001, MNRAS, 326. 1067

Pearson K.. Horne K. \& Skidmore W., 2003. MNRAS. 338,1067

Pearson K., Horne K. \& Skidmore W., 200.4. MNR.tS. in prep.

Skidmore W., Pearson K., O'Brien K., Horne K.. Gomer R. \& Oke B., 2003a, MNRAS, 338. 1057

Skidmore W., Gomer R., Horne K., O'Brien K., Oke B. \& Pearson K., 2003b, Proc. IAU Coll. 190, Pub. ASP Conf. series, in press, Eds. M. Cropper, S. Vrielmann

Schwarz R., Hedelt P., Rau A., Staude A., Schwope A.. 2002, ASP Conf. Proc., 261, 167

Steeghs D., O'Brien K., Horne K., Gomer R. \& Oke B., 2001, MNRAS, 323, 484

Richard Gomer: Howard Hughes Medical Institute, Department of Biochemistry \& Cell Biology, MS-140, Rice University, Houston, TX 77005-1892, USA (richard (1)ioc.rice.edu).

Keith Horne: School of Physics \& Astronomy, University of St. Andrews, North Haugh. St. Andrew's. Fife. KY16 9SS, UK (kdh1@st-andrews.ac.uk).

Kieran O'Brien: ESO Headquarters Chile office, Alonso de Córdova 3107, Vitacura, Casilla 19001. Santiago 19, Chile(kobrien@eso.org).

Bev Oke: Dominion Astrophysical Observatory, 5071 West Saanich Road, Victoria, BC (bev.oke (inrc.ca).

Kevin Pearson: Louisiana State University, Department of Physics \& Astronomy, 202 Nicholson Hall. Tower Drive, Baton Rouge, Louisiana 70803-4001, USA (kjp1@baton.phys.lsu.edu).

Warren Skidmore: 105-24 Caltech, 1201 East California Blvd., Pasadena, CA 91125. USA (was ciast ro.caltech. edu). 